\title{
Appropriate Polypharmacy and Medicine Safety: When Many is not Too Many
}

\author{
Cathal A. Cadogan ${ }^{1,2}$ - Cristín Ryan ${ }^{1,2}$ - Carmel M. Hughes ${ }^{1}$
}

Published online: 21 December 2015

(C) The Author(s) 2015. This article is published with open access at Springerlink.com

\begin{abstract}
The use of multiple medicines (polypharmacy) is increasingly common in middle-aged and older populations. Ensuring the correct balance between the prescribing of 'many' drugs and 'too many' drugs is a significant challenge. Clinicians are tasked with ensuring that patients receive the most appropriate combinations of medications based on the best available evidence, and that medication use is optimised according to patients' clinical needs (appropriate polypharmacy). Historically, polypharmacy has been viewed negatively because of the associated medication safety risks, such as drug interactions and adverse drug events. More recently, polypharmacy has been identified as a risk factor for under-prescribing, such that patients do not receive necessary medications and this can also pose risks to patients' safety and well-being. The negative connotations that have long been associated with the term polypharmacy could potentially be acting as a driving factor for under-prescribing, whereby clinicians are reluctant to prescribe necessary medicines for patients who are already receiving 'many' medicines. It is now recognised that the prescribing of 'many' medicines can be entirely appropriate in patients with several chronic con-
\end{abstract}

Cathal A. Cadogan

cathalcadogan@rcsi.ie

1 School of Pharmacy, Queen's University Belfast, Belfast, UK

2 RCSI School of Pharmacy, Royal College of Surgeons in Ireland, Ardilaun House, Block B, 111 St Stephen's Green, Dublin 2, Ireland ditions and that the risks of adverse drug events that have been associated with polypharmacy may be greatly reduced when patients' clinical context is taken into consideration. In this article, we outline the current perspectives on polypharmacy and make the case for adopting the term 'appropriate polypharmacy' in differentiating between the prescribing of 'many' drugs and 'too many' drugs. We also outline the inherent challenges in doing so and provide recommendations for future clinical practice and research.

\section{Key points}

Despite the fact that the prescribing of multiple medicines (polypharmacy) is increasingly common in middle-aged (age 45-64 years) and older populations (age $\geq 65$ years), the term 'polypharmacy' continues to lack a universally accepted definition.

Recent cohort studies using population datasets have challenged long-standing assumptions that polypharmacy is always hazardous, or indicative of poor care, and have highlighted the importance of considering the clinical context underlying prescribing.

The concept of 'appropriate polypharmacy' recognises that patients can benefit from multiple medications provided that prescribing is evidence based, reflects patients' clinical conditions and considers potential drug interactions. This concept should be promoted in place of existing thresholds that define the term 'polypharmacy' using an arbitrary number of medicines. 


\section{Introduction}

The prescribing of multiple medicines, or polypharmacy, is increasingly common in clinical practice, particularly in older people (age $\geq 65$ years) [1-3]. This is likely owing to multiple factors including increasing life expectancy and the consequent growth in the prevalence of multimorbidity (i.e. the presence of two or more long-term conditions), as well as the enhanced availability of effective drug treatments and prescribing guidelines that advocate the use of more than one drug in the prevention and management of various health conditions $[4,5]$.

Polypharmacy is often clinically indicated and beneficial in specific conditions (e.g. diabetes mellitus, hypertension) and patient populations (e.g. patients with multimorbidity). However, the use of multiple medicines poses medication safety risks to patients $[5,6]$. For example, a repeated cross-sectional analysis of community-dispensed prescribing data for 310,000 adults in Scotland found that the number of dispensed medications was the characteristic most strongly associated with potentially serious drug-drug interactions; $81 \%$ of patients receiving $\geq 15$ drugs were exposed to potentially serious interactions compared with $11 \%$ of those dispensed two to four drugs [5]. Drug interactions are a form of adverse drug event (ADE) that have been identified as a preventable cause of medicationrelated hospitalisations [7, 8]. Balancing the clinical benefits and safety risks associated with the use of multiple medications is a difficult task; hence, polypharmacy has been described as one of the greatest prescribing challenges [9].

This article outlines current perspectives on polypharmacy, makes the case for adopting the term 'appropriate polypharmacy' in ensuring patient safety, and outlines the inherent challenges for practice and research in doing so.

\section{Polypharmacy: 'Many' Medicines or 'Too Many' Medicines?}

One of the challenges in discussing polypharmacy and the associated medication safety implications is that the term itself currently lacks a universally accepted definition. Although we have referred to polypharmacy as the prescribing of multiple medicines, it is also commonly defined using a numerical threshold (e.g. co-prescribing of four or five medications) $[10,11]$. The term can also have a dual meaning $[12,13]$. For example, polypharmacy can refer to the prescribing of 'many drugs' or 'too many' drugs, the former being entirely appropriate [12]. Polypharmacy has often been viewed negatively and seen to signify inappropriate drug therapy or 'too many' drugs [12, 13]. Observational studies have highlighted the association between polypharmacy in older people and potentially inappropriate prescribing (PIP) [14, 15], as well as negative clinical outcomes that pose risks to patients' safety and well-being (e.g. ADEs, medication non-adherence) [11]. Hence, interventions aimed at improving the appropriateness of prescribing for patients receiving polypharmacy have often focussed on reducing the number of prescribed medications [16].

However, interventions that seek to solely reduce the number of prescribed medicines fail to consider inappropriate prescribing in its entirety. Inappropriate prescribing does not only encompass over-prescribing (prescribing of more drugs than clinically necessary) and mis-prescribing (incorrect prescribing of a necessary drug), it also includes under-prescribing (failure to prescribe a clinically indicated drug) [17, 18]. Under-prescribing is common in older patients and the associated clinical consequences pose safety risks to patients (e.g. ischaemic stroke in patients undertreated for atrial fibrillation) [19]. Polypharmacy has been associated with under-prescribing in older people [20, 21]. For example, Kuijpers et al. [20] showed that a significantly higher proportion of patients receiving polypharmacy (defined as four or more medicines) were under-treated $(42.9 \%)$ compared with those receiving fewer medicines $(13.5 \%)$ ). It has been speculated that fear of causing ADEs is contributing to under-prescribing by clinicians [19] and this could partly account for the reported reluctance among clinicians to initiate new medications in patients receiving polypharmacy [22].

Recent cohort studies using population datasets have challenged existing assumptions that polypharmacy is always hazardous, or indicative of poor care, and have highlighted the importance of considering the clinical context underlying prescribing [23, 24]. For example, an analysis of Scottish primary care data linked to hospitalisations for a sample of approximately 180,000 adults showed that the strength of the association between the number of regular medicines and unplanned hospitalisations was greatly reduced when the number of clinical conditions was accounted for [23]. As noted by Guthrie et al. [5] polypharmacy is "potentially problematic rather than always inappropriate" and, as such, assessments of prescribing appropriateness need to extend beyond the number of drugs prescribed and consider co-existing medical conditions in differentiating between 'many' and 'too many' medicines.

Owing to the lack of a universally accepted definition, there have been calls to abandon the term polypharmacy in favour of terms such as 'hyperpharmacotherapy' or 'polytherapy' $[13,25]$. However, it is difficult to see this happening because of the term's widespread use in the literature. Instead, an arguably better solution to this longstanding issue would be to follow Aronson's 
recommendation and qualify the term as either appropriate or inappropriate [12]. A recent report published by the King's Fund in the UK has promoted the term 'appropriate polypharmacy', described as 'prescribing for an individual with complex or multiple conditions where medicine use has been optimised and prescribing is in accordance with best evidence', rather than existing thresholds that define polypharmacy based on the number of prescribed medications [26]. The concept of 'appropriate polypharmacy' recognises that patients can benefit from multiple medications provided that prescribing is evidence based and reflects patients' clinical needs. Greater use of this term may help to dispel existing misconceptions that polypharmacy signifies 'too many' medicines and reduce reluctance among clinicians to initiate necessary medicines in patients already receiving polypharmacy.

\section{Challenges of Ensuring Appropriate Polypharmacy}

Widespread adoption of the term 'appropriate polypharmacy' is by no means straightforward. Considerable challenges need to be overcome to enable its use by both researchers and clinicians in terms of establishing methods for identifying patients at risk of PIP and operationalising the core concepts of appropriate polypharmacy in clinical practice, i.e. evidence-based prescribing and medicines optimisation.

\subsection{Identifying Patients with Medication Safety Risks}

The use of numerical thresholds as a screening tool for polypharmacy has long provided a relatively straightforward method for identifying patients deemed at risk of PIP or ADEs. However, the validity of this approach appears to be questionable [27-29]. For example, using a cohort of older trial participants, Belfrage et al. [27] examined the concurrent validity of the number of prescribed drugs as an indicator of prescribing quality (i.e. ability to differentiate between appropriate and suboptimal treatment). The authors showed that a cut-off indicator of five or more medicines had acceptable sensitivity (14\% of patients receiving suboptimal treatment were not identified) but poor specificity (47\% of identified patients were not receiving suboptimal treatment) in detecting PIP using a validated assessment tool as the gold standard (i.e. STOPP/ START [30]). They also identified an inverse relationship between the parameters of sensitivity and specificity. Sensitivity was found to decline rapidly when a larger number of medications were used as a cut-off indicator whereby higher proportions of patients requiring treatment optimisation would not be identified (Fig. 1).

The King's Fund report proposed a pragmatic approach for identifying patients with 'higher-risk polypharmacy' (e.g. $\geq 10$ medications), noting, as already stated, that there is no ideal and universally applicable definition for identifying polypharmacy [26]. In cases where numerical thresholds are used, it must be recognised that a proportion of patients receiving inappropriate medications will be overlooked (Fig. 1). A targeted approach, focussing on specific drug classes that contribute to PIP, has been proposed as a more suitable method that may provide greater sensitivity in identifying patients with medication safety risks [13, 29].

\subsection{Polypharmacy and Appropriate Prescribing}

Ensuring that prescribing is evidence based is one of the key challenges to achieving appropriate polypharmacy, particularly in older people and patients with multimorbidity. It is well recognised that evidence to support prescribing decisions in older people is lacking because of their under-representation in clinical trials [31]. Furthermore, prescribing guidelines typically focus on single diseases and when applied to complex multimorbid patients, they often fail to provide guidance on how to prioritise treatment recommendations and can act as a driving force for polypharmacy [32].

A number of recent developments have sought to address some of these issues. For example, a working group in Scotland has published guidance that outlines a seven-step structured process for conducting medication reviews with patients receiving polypharmacy [33]. An expert panel from the American Geriatrics Society has developed a set of guiding principles on the management of older patients with multimorbidity [34] (Fig. 2). These guidance sources outline a patient-centred approach to ensuring safe and appropriate medicine use. In addition to these resources, the National Institute for Health and Care Excellence in the UK is currently developing guidelines for the clinical assessment and management of patients with multimorbidity [35]. However, it will be some time before the clinical impact of these resources is known.

Various tools have been developed to assess prescribing appropriateness, primarily in older populations [18]. These assessment tools have typically been developed using consensus-based exercises (e.g. Delphi panels), to formulate an evidence base where higher levels of evidence (e.g. controlled trials) are lacking [18]. Although the current literature on polypharmacy primarily focuses on the older population, the use of multiple medicines is by no means limited to older people. Multimorbidity is also highly prevalent in middle-aged people (age 45-64 years) [36]. 
Fig. 1 Plot of cut-off indicator vs sensitivity and specificity (adapted from Belfrage et al. [27])
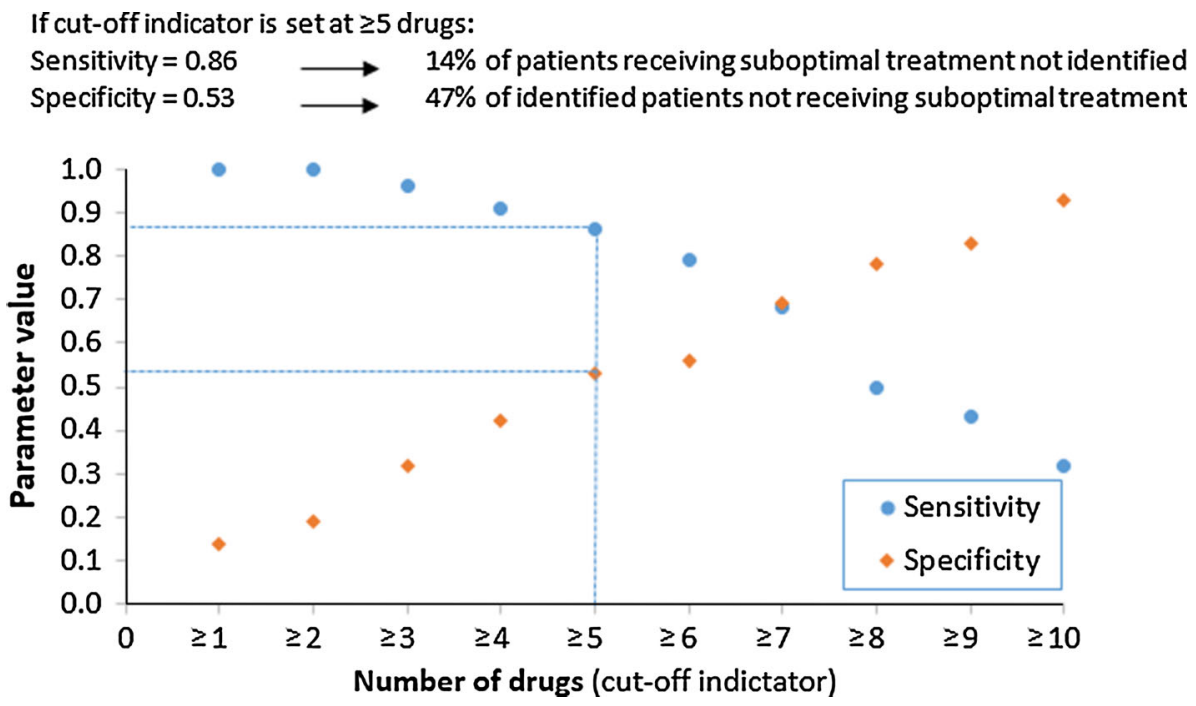

Despite a dearth of prescribing evaluations among this population cohort [37], emerging evidence shows that polypharmacy is increasingly common in middle-aged people [5, 38], thus, exposing them to medication safety risks. A set of prescribing criteria known as PROMPT (PRescribing Optimally in Middle-aged People's Treatments) has been developed specifically for use in middleaged adults [37].

Prescribing assessment tools can play an important role in identifying and addressing PIP. However, there are limitations associated with their clinical application in ensuring appropriate polypharmacy. For example, they do not provide guidance as to how treatment decisions should be prioritised and in many cases, predictive validity has not been established [39]. Therefore, the clinical impact of improvements in prescribing appropriateness on patient safety is unclear. Furthermore, as there is no universal indicator of appropriate polypharmacy, these tools act as surrogate markers and additional amendments may be required to ensure that prescribing is appropriate for individual patients.

\subsection{Polypharmacy and Medicines Optimisation}

Another key challenge to achieving appropriate polypharmacy relates to optimising patients' medicine use. Medicines optimisation is a person-centred approach aimed at ensuring the best clinical outcomes for patients through safe and effective use of medicines [40]. Medicines optimisation centres on shared decision making that considers an individual patient's needs, preferences, and values. Optimising polypharmacy involves "encouraging the use of appropriate drugs, in a way that the patient is willing and able to comply with, to treat the right diseases", as well as targeting both over-prescribing and under-prescribing [26].
Efforts to optimise polypharmacy often focus on targeting over-prescribing, thereby reducing treatment burden. This is exemplified by the emergence of the term 'deprescribing' that is featuring increasingly in the literature. Deprescribing has been defined as "the systematic process of identifying and discontinuing drugs in instances in which existing or potential harms outweigh existing or potential benefits within the context of an individual patient's care goals, current level of functioning, life expectancy, values, and preferences" [41]. Deprescribing has been described as a patient-centred process that forms part of the good prescribing continuum and has been promoted as a strategy for reducing polypharmacy and improving patients outcomes [41-43]. However, it is important to note that deprescribing deals with only one aspect of inappropriate prescribing (i.e. over-prescribing). Although it has been postulated that deprescribing may indirectly serve to reduce under-prescribing [42], this has not been established. More importantly, evidence to support the entire process is lacking and claims of benefits are often based on indirect evidence and inferences from previous research involving older people [44, 45]. Furthermore, a recent systematic review of observational studies that examined clinical outcomes in community-dwelling older patients receiving multiple medicines, found that evidence was mixed regarding the relationship between polypharmacy and adverse outcomes such as falls, hospitalisations and mortality [46]. Thus, the associations between polypharmacy and negative clinical outcomes might not be as well established as previously believed, particularly because many studies did not adequately account for comorbidity as a confounding factor. It is clear that if appropriate polypharmacy is to be achieved, deprescribing cannot be considered in isolation when optimising patients' medications, particularly as potentially 
Fig. 2 Structured approach for evaluating and managing older adults with multimorbidity (adapted from the American Geriatrics Society [34])

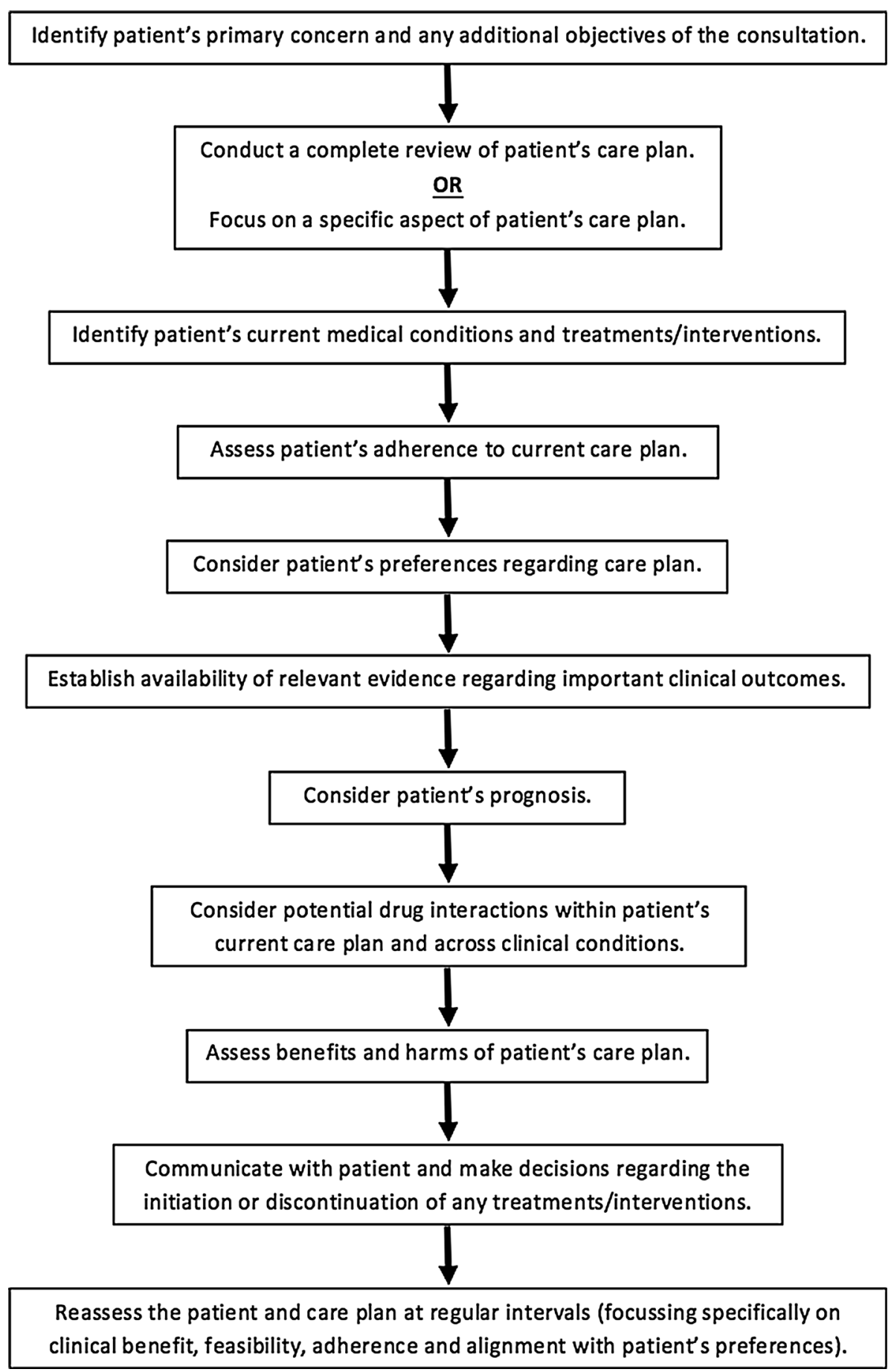

inappropriate omissions have been found to be more prevalent than potentially inappropriate prescriptions in older patients [21]. Notwithstanding the above limitations, the process of optimising polypharmacy could benefit from elements of the deprescribing process (e.g. systematic approach to medication review, addition of expiration dates to medication regimens to prompt reviews) [41].
In optimising polypharmacy, it is also important to note that efforts to ensure safe and effective medicine use are not determined solely by the number of medications prescribed. Additional factors contribute to the overall complexity of drugs regimens (i.e. formulation types, dosing frequency, further directions). These factors have been incorporated into the Medication Regimen Complexity 
Index, a validated tool that can be used to quantify drug regimen complexity [47]. Despite being more time consuming to apply than simple medication counts, this index could encourage greater consideration of important and modifiable factors (e.g. dosing frequency, additional directions) other than the number of medications in optimising polypharmacy. This may also help to ensure that patients are willing and able to adhere to the prescribed medication regimen. Medication adherence is an important clinical issue in ensuring safe and effective medicine use as it has been estimated that $50 \%$ of patients do not take their medications correctly [48]. Polypharmacy is associated with medication non-adherence in older people [11] and although few studies have assessed the full range of factors that contribute to medication regimen complexity, higher Medication Regimen Complexity Index scores have been associated with lower adherence in older patients [49]. Few trials of interventions seeking to improve appropriate polypharmacy in older people have examined patient adherence as an outcome measure [10]. However, there is some evidence to suggest that regimen simplification (e.g. reduction in dosing frequency) may be an important effect moderator for interventions aimed at improving adherence in this patient cohort [50].

\section{Recommendations for Future Clinical Practice and Research}

In ensuring the prescribing of appropriate polypharmacy, researchers and clinicians need to maintain a broad focus. The challenges posed by the prescribing of polypharmacy are neither limited to the older population nor the prescribing of 'too many' drugs. In the absence of a universally applicable, valid and reliable measure of appropriate polypharmacy, future assessments of prescribing appropriateness should assess both under-prescribing and overprescribing across all adult populations using validated tools as surrogate markers (e.g. STOPP/START [51], PROMPT [37]). Medication regimen complexity should also be considered to reduce factors other than the absolute number of medications that contribute to the overall treatment burden (e.g. dosing frequency). This could ultimately enhance medication adherence.

Efforts to optimise polypharmacy should adopt a patient-centred approach as advocated by recent guidance [33, 34]. It is important to recognise that appropriate polypharmacy is not a fixed end-point but rather an ideal concept. The threshold that differentiates between the prescribing of 'many' drugs and 'too many' drugs will not only vary according to individual patient's clinical conditions, but also over time. For example, as multimorbid patients age and become frail, preventive medications become less meaningful, and clinicians must establish when it is appropriate to transition from disease-modifying treatment to a palliative approach, whereby medications are reduced or discontinued [26]. Accordingly, regular medication reviews will serve an increasingly important function in ensuring appropriate prescribing according to patients' existing clinical conditions and life expectancy.

In the absence of a universal indicator with suitable sensitivity and specificity for identifying patients receiving inappropriate polypharmacy, it may be better to focus on specific drug classes that pose safety risks. Researchers and clinicians can draw from the existing body of observational research on PIP to identify suitable target drug classes.

Finally, more attention needs to be paid to the clinical outcomes associated with appropriate polypharmacy. A Cochrane review of interventions to improve appropriate polypharmacy in older people highlighted that assessments of clinically relevant outcomes (e.g. quality of life) have been lacking in trials to date and, where assessed, findings have often been inconsistent or difficult to compare because of heterogeneity in measurement scales [10]. Increasing emphasis is being placed on the importance of 'core outcome sets' (COS) as an agreed and standardised set of outcomes that should be measured and reported, as a minimum, in all trials in a specific clinical area [52]. COS are intended to ensure that selected outcomes are relevant to key stakeholders and to overcome problems with heterogeneity in outcome measurements that have hindered the pooling of data in systematic reviews. Ongoing work as part of the COMET (Core Outcome Measures in Effectiveness Trials) initiative is seeking to establish rigorous methods for developing COS $[52,53]$ and future research should focus on developing a COS for trials of interventions aimed at improving appropriate polypharmacy.

\section{Conclusion}

In current clinical practice where the prescribing of multiple medicines is increasingly common in adults with multimorbidity and advanced age, differentiating between 'many' drugs and 'too many' drugs is proving ever more complex. Previous assumptions that polypharmacy is synonymous with 'too many' medicines or inappropriate prescribing have been undermined because when patients' clinical context is taken into consideration, the prescribing of 'many' medicines can, in fact, be entirely appropriate. Conceptualising polypharmacy as a numerical threshold is unhelpful because it fails to consider that the appropriate number of medicines varies according to individual patients' clinical needs and may overlook the omission of potentially beneficial medications, which can equally pose 
risks to patients' safety and well-being. We contend that, as a stand-alone term, polypharmacy should be interpreted as the prescribing of multiple medicines and, rather than quantifying the term according to the number of prescribed medicines, greater emphasis should be placed on qualifying the term based on the clinical appropriateness of the combination of medicines for the individual patient. Increased use of the term 'appropriate polypharmacy' could encourage greater consideration of the clinical context underlying prescribing, as well as increased acceptance that the prescribing of multiple medicines is 'potentially problematic rather than always inappropriate'.

\section{Compliance with Ethical Standards}

Funding During the writing of this paper, C. A. Cadogan was being supported by a project grant from the Dunhill Medical Trust (Grant No. R298/0513), focusing on the development of an intervention to improve appropriate polypharmacy in older people in primary care.

Conflict of interest Cathal Cadogan, Cristín Ryan and Carmel Hughes have no conflicts of interest to declare that are directly relevant to the content of this manuscript.

Open Access This article is distributed under the terms of the Creative Commons Attribution-NonCommercial 4.0 International License (http://creativecommons.org/licenses/by-nc/4.0/), which permits any noncommercial use, distribution, and reproduction in any medium, provided you give appropriate credit to the original author(s) and the source, provide a link to the Creative Commons license, and indicate if changes were made.

\section{References}

1. Sumukadas D, McMurdo ME, Mangoni AA, Guthrie B. Temporal trends in anticholinergic medication prescription in older people: repeated crosssectional analysis of population prescribing data. Age Ageing. 2014;43(4):515-21.

2. Hovstadius B, Hovstadius K, Astrand B, Petersson G. Increasing polypharmacy: an individual-based study of the Swedish population 2005-2008. BMC Clin Pharmacol. 2010;10:16.

3. Franchi C, Tettamanti M, Pasina L, Djignefa CD, Fortino I, Bortolotti A, et al. Changes in drug prescribing to Italian community-dwelling elderly people: The EPIFARM-Elderly Project 2000-2010. Eur J Clin Pharmacol. 2014;70(4):437-43.

4. Hughes C, Cooper JA, Ryan C. Going beyond the numbers: a call to redefine polypharmacy. $\mathrm{Br} \mathrm{J}$ Clin Pharmacol. 2014;77(6):915-6.

5. Guthrie B, Makubate B, Hernandez-Santiago V, Dreischulte T. The rising tide of polypharmacy and drug-drug interactions: population database analysis 1995-2010. BMC Med. 2015;13:74.

6. Akbarov A, Kontopantelis E, Sperrin M, Stocks SJ, Williams R, Rodgers S, et al. Primary care medication safety surveillance with integrated primary and secondary care electronic health records: a cross-sectional study. Drug Saf. 2015;38(7):671-82.

7. Juurlink DN, Mamdani M, Kopp A, Laupacis A, Redelmeier DA. Drug-drug interactions among elderly patients hospitalized for drug toxicity. JAMA. 2003;289(13):1652-8.

8. Leendertse AJ, Egberts ACG, Stoker LJ, van den Bemt PMLA. Frequency of and risk factors for preventable medication-related hospital admissions in the Netherlands. Arch Intern Med. 2008;168(17):1890-6.

9. Payne RA, Avery AJ. Polypharmacy: one of the greatest prescribing challenges in general practice. $\mathrm{Br} \mathrm{J}$ Gen Pract. 2011;61(583):83-4.

10. Patterson S, Cadogan C, Kerse N, Cardwell C, Bradley M, Ryan $\mathrm{C}$, et al. Interventions to improve the appropriate use of polypharmacy for older people. Cochrane Database Syst Rev. 2014;10:CD008165.

11. Maher RL, Hanlon J, Hajjar ER. Clinical consequences of polypharmacy in elderly. Expert Opin Drug Saf. 2014;13(1):57-65.

12. Aronson JK. In defence of polypharmacy. Br J Clin Pharmacol. 2004;57(2):119-20.

13. Bushardt RL, Massey EB, Simpson TW, Ariail JC, Simpson KN. Polypharmacy: misleading, but manageable. Clin Interv Aging. 2008;3(2):383-9.

14. Cahir C, Fahey T, Teeling M, Teljeur C, Feely J, Bennett K. Potentially inappropriate prescribing and cost outcomes for older people: a national population study. $\mathrm{Br} \mathrm{J}$ Clin Pharmacol. 2010;69(5):543-52.

15. Bradley MC, Fahey T, Cahir C, Bennett K, O’Reilly D, Parsons $\mathrm{C}$, et al. Potentially inappropriate prescribing and cost outcomes for older people: a cross-sectional study using the Northern Ireland Enhanced Prescribing Database. Eur J Clin Pharmacol. 2012;68(10):1425-33.

16. Rollason V, Vogt N. Reduction of polypharmacy in the elderly: a systematic review of the role of the pharmacist. Drugs Aging. 2003;20(11):817-32.

17. Spinewine A, Schmader KE, Barber N, Hughes C, Lapane KL, Swine C, et al. Appropriate prescribing in elderly people: how well can it be measured and optimised? Lancet. 2007;370(9582):173-84.

18. Kaufmann CP, Tremp R, Hersberger KE, Lampert ML. Inappropriate prescribing: a systematic overview of published assessment tools. Eur J Clin Pharmacol. 2014;70(1):1-11.

19. Cherubini A, Corsonello A, Lattanzio F. Underprescription of beneficial medicines in older people. Drugs Aging. 2012;29(6):463-75.

20. Kuijpers MAJ, van Marum RJ, Egberts ACG, Jansen PAF. Relationship between polypharmacy and underprescribing. Br J Clin Pharmacol. 2008;65(1):130-3.

21. Galvin R, Moriarty F, Cousins G, Cahir C, Motterlini N, Bradley $\mathrm{M}$, et al. Prevalence of potentially inappropriate prescribing and prescribing omissions in older Irish adults: findings from The Irish LongituDinal Study on Ageing study (TILDA). Eur J Clin Pharmacol. 2014;70(5):599-606.

22. Hancock HC, Close H, Fuat A, Murphy JJ, Hungin APS, Mason $\mathrm{JM}$. Barriers to accurate diagnosis and effective management of heart failure have not changed in the past 10 years: a qualitative study and national survey. BMJ Open. 2014;4(3):e003866.

23. Payne RA, Abel GA, Avery AJ, Mercer SW, Roland MO. Is polypharmacy always hazardous? A retrospective cohort analysis using linked electronic health records from primary and secondary care. Br J Clin Pharmacol. 2014;77(6):1073-82.

24. Appleton SC, Abel GA, Payne RA. Cardiovascular polypharmacy is not associated with unplanned hospitalisation: evidence from a retrospective cohort study. BMC Fam Pract. 2014;15:58.

25. Lopez-Briz E, Ruiz-Garcia V, Carbonell-Sanchis R. Re: Polypharmacy: a necessary evil. BMJ. 2013;347:f7033.

26. The King's Fund. Polypharmacy and medicines optimisation: making it safe and sound. London; 2013. Available from: http:// www.kingsfund.org.uk/sites/files/kf/field/field_publication_file/ polypharmacy-and-medicines-optimisation-kingsfund-nov13.pdf. Accessed 13 Dec 2015. 
27. Belfrage B, Koldestam A, Sjöberg C, Wallerstedt SM. Number of drugs in the medication list as an indicator of prescribing quality: a validation study of polypharmacy indicators in older hip fracture patients. Eur J Clin Pharmacol. 2015;71(3):363-8.

28. Viktil KK, Blix HS, Moger TA, Reikvam A. Polypharmacy as commonly defined is an indicator of limited value in the assessment of drug-related problems. $\mathrm{Br} \mathrm{J}$ Clin Pharmacol. 2007;63(2):187-95.

29. Carey IM, De Wilde S, Harris T, Victor C, Richards N, Hilton $\mathrm{SR}$, et al. What factors predict potentially inappropriate primary care prescribing in older people? Analysis of UK primary care patient record database. Drugs Aging. 2008;25(8):693-706.

30. Gallagher P, Ryan C, Byrne S, Kennedy J, O'Mahony D. STOPP (Screening Tool of Older Person's Prescriptions) and START (Screening Tool to Alert doctors to Right Treatment). Consensus validation. Int J Clin Pharmacol Ther. 2008;46(2):72-83.

31. Broekhuizen K, Pothof A, de Craen AJM, Mooijaart SP. Characteristics of randomized controlled trials designed for elderly: a systematic review. PLoS One. 2015;10:e126709.

32. Hughes LD, McMurdo MET, Guthrie B. Guidelines for people not for diseases: the challenges of applying UK clinical guidelines to people with multimorbidity. Age Ageing. 2013;42(1):62-9.

33. Scottish Government Model of Care Polypharmacy Working Group. Polypharmacy guidance (2nd edition). Scotland; 2015. Available from: http://www.sign.ac.uk/pdf/polypharmacy_ guidance.pdf. Accessed 13 Dec 2015.

34. Ickowicz E. Patient-centered care for older adults with multiple chronic conditions: a stepwise approach from the American Geriatrics society. J Am Geriatr Soc. 2012;60(10):1957-68.

35. National Institute for Health and Care Excellence. Multimorbidity: assessment, prioritisation and management of care for people with commonly occurring multimorbidities. Available from: https://www.nice.org.uk/guidance/indevelopment/gidcgwave0704. Accessed 13 Dec 2015.

36. Barnett K, Mercer SW, Norbury M, Watt G, Wyke S, Guthrie B. Epidemiology of multimorbidity and implications for health care, research, and medical education: a cross-sectional study. Lancet. 2012;380(9836):37-43.

37. Cooper JA, Ryan C, Smith SM, Wallace E, Bennett K, Cahir C, et al. The development of the PROMPT (PRescribing Optimally in Middle-aged People's Treatments) criteria. BMC Health Serv Res. 2014;14:484.

38. Moriarty F, Hardy C, Bennetta K, Smith S, Fahey T. Trends in polypharmacy and prescribing appropriateness from 1997 to 2012. Int J Pharm Pract. 2015;23(S1):24-5.

39. Cahir C, Moriarty F, Teljeur C, Fahey T, Bennett K. Potentially inappropriate prescribing and vulnerability and hospitalization in older community-dwelling patients. Ann Pharmacother. 2014;48(12):1546-54.

40. National Institute for Health and Care Excellence. NICE guidelines [NG5]: medicines optimisation: the safe and effective use of medicines to enable the best possible outcomes. United Kingdom; 2015. Available from: https://www.nice.org.uk/guidance/ng5. Accessed 13 Dec 2015.

41. Scott IA, Hilmer SN, Reeve E, Potter K, Le Couteur D, Rigby D, et al. Reducing inappropriate polypharmacy: the process of deprescribing. JAMA Intern Med. 2015;175(5):827-34.

42. Reeve E, Shakib S, Hendrix I, Roberts MS, Wiese MD. The benefits and harms of deprescribing. Med $\mathrm{J}$ Aust. 2014;201(7):386-9.

43. Scott IA, Anderson K, Freeman CR, Stowasser DA. First do no harm: a real need to deprescribe in older patients. Med J Aust. 2014;201(7):390-2.

44. Reeve E, Shakib S, Hendrix I, Roberts MS, Wiese MD. Review of deprescribing processes and development of an evidencebased, patient-centred deprescribing process. Br J Clin Pharmacol. 2014;78(4):738-47.

45. Reeve E, Wiese MD. Benefits of deprescribing on patients' adherence to medications. Int J Clin Pharm. 2014;36(1):26-9.

46. Fried TR, O'Leary J, Towle V, Goldstein MK, Trentalange M, Martin DK. Health outcomes associated with polypharmacy in community-dwelling older adults: a systematic review. J Am Geriatr Soc. 2014;62(12):2261-72.

47. George J, Phun YT, Bailey MJ, Kong DCM, Stewart K. Development validation of the medication regimen complexity index. Ann Pharmacother. 2004;38(9):1369-76.

48. De Cates Angharad N, Farr Matthew RB, Wright N, Jarvis Morag C, Rees K, Ebrahim S, et al. Fixed-dose combination therapy for the prevention of cardiovascular disease. Cochrane Database Syst Rev. 2014;4:CD009868.

49. Mansur N, Weiss A, Beloosesky Y. Looking beyond polypharmacy: quantification of medication regimen complexity in the elderly. Am J Geriatr Pharmacother. 2012;10(4):223-9.

50. Conn VS, Hafdahl AR, Cooper PS, Ruppar TM, Mehr DR, Russell CL. Interventions to improve medication adherence among older adults: meta-analysis of adherence outcomes among randomized controlled trials. Gerontologist. 2009;49(4):447-62.

51. O'Mahony D, O'Sullivan D, Byrne S, O'Connor MN, Ryan C, Gallagher P. STOPP/START criteria for potentially inappropriate prescribing in older people: version 2. Age Ageing. 2014;44(2):213-8.

52. Williamson PR, Altman DG, Blazeby JM, Clarke M, Devane D, Gargon E, et al. Developing core outcome sets for clinical trials: issues to consider. Trials. 2012;13:132.

53. Prinsen CAC, Vohra S, Rose MR, King-Jones S, Ishaque S, Bhaloo Z, et al. Core Outcome Measures in Effectiveness Trials (COMET) initiative: protocol for an international Delphi study to achieve consensus on how to select outcome measurement instruments for outcomes included in a "core outcome set". Trials. 2014; 15:247. 